\title{
On periodic solutions of a class of $k$-dimensional systems of max-type difference equations
}

\section{Stevo Stević}

\author{
"Correspondence: sstevic@ptt.rs \\ Mathematical Institute of the \\ Serbian Academy of Sciences, Knez \\ Mihailova 36/III, Beograd, 11000 , \\ Serbia \\ Operator Theory and Applications \\ Research Group, Department of \\ Mathematics, King Abdulaziz \\ University, P.O. Box 80203, Jeddah, \\ 21589, Saudi Arabia
}

\begin{abstract}
Some sufficient conditions are given such that the following system of difference equations:

$$
\begin{aligned}
& x^{(1)}(n+1)=\max _{1 \leq j \leq l_{1}}\left\{f_{1 j}\left(n, x^{(1)}(n), \ldots, x^{(k)}(n)\right)\right\}, \\
& x^{(2)}(n+1)=\max _{1 \leq j \leq l_{2}}\left\{f_{2 j}\left(n, x^{(1)}(n), \ldots, x^{(k)}(n)\right)\right\}, \\
& \vdots \\
& x^{(k)}(n+1)=\max _{1 \leq j \leq l_{k}}\left\{f_{k j}\left(n, x^{(1)}(n), \ldots, x^{(k)}(n)\right)\right\},
\end{aligned}
$$

$n \in \mathbb{N}_{0}$, where $k \in \mathbb{N}, l_{i} \in \mathbb{N}, i=\overline{1, k}, x^{(i)}(0) \in \mathbb{R}, i=\overline{1, k}$, has a unique periodic solution attracting all the solutions to the system. Our main result considerably generalizes some recent results in the literature and simplifies their proofs.
\end{abstract}

MSC: Primary 39A23

Keywords: max-type system of difference equations; existence of periodic solutions; global attractivity

\section{Introduction}

Max-type difference equations and systems have attracted some recent interest (see, for example, [1-17], and the references therein). Besides the usual interest in mathematics, one of the reasons for the interest is their potential application in control theory, neuroscience, and some other branches of science, which is under present investigation of some experts in the research fields. The problem of calculating the maximal output among several possible ones suggests a great applicable potential whenever there is such a situation in applications and/or theory. The boundedness character of, usually positive, solutions of the equations and systems is frequently studied as one of the basic problems in this area (see, for example, $[1,6,7,9,11,15]$ ). The study of concrete symmetric and close to symmetric systems of difference equations essentially stems [18] and [19] by Papaschinopoulos and Schinas. The investigation of max-type systems in this direction was conducted, for example, in [6-8, 13-16]. Many papers on max-type difference equations and systems deal with

(c) 2016 Stević. This article is distributed under the terms of the Creative Commons Attribution 4.0 International License (http://creativecommons.org/licenses/by/4.0/), which permits unrestricted use, distribution, and reproduction in any medium, provided you give appropriate credit to the original author(s) and the source, provide a link to the Creative Commons license, and indicate if changes were made. 
the problem of periodicity of their solutions (see, for example, $[1,3,4,7,12,13,16,17]$, and the references therein), while global attractivity results can be found, for example, in [2, 5, $10,14]$. For the solvability of some max-type systems see [20] (there has been a renewed interest in the topic, see, also [21] and numerous references therein). The majority of the above mentioned papers are on concrete max-type systems of difference equations, but there are a few dealing with some general systems, such as $[13,16]$, which are motivated by important note [12].

As usual, throughout the paper, $\mathbb{N}$ will denote the set of all natural numbers, i.e., the set $\{1,2,3, \ldots\}, \mathbb{N}_{0}=\mathbb{N} \cup\{0\}$, and $\mathbb{R}$ will denote the set of all real numbers. If $k, l \in \mathbb{N}_{0}$ are such that $k \leq l$, then the notation $i=\overline{k, l}$ will denote the set of all $i \in \mathbb{N}_{0}$ such that $k \leq i \leq l$.

In this paper we consider the following, quite general, max-type system of difference equations extending the one in [2] and related to the ones in $[13,16]$ :

$$
\begin{aligned}
& x^{(1)}(n+1)=\max _{1 \leq j \leq l_{1}}\left\{f_{1 j}\left(n, x^{(1)}(n), \ldots, x^{(k)}(n)\right)\right\}, \\
& \vdots \\
& x^{(i)}(n+1)=\max _{1 \leq j \leq l_{i}}\left\{f_{i j}\left(n, x^{(1)}(n), \ldots, x^{(k)}(n)\right)\right\}, \\
& \vdots \\
& x^{(k)}(n+1)=\max _{1 \leq j \leq l_{k}}\left\{f_{k j}\left(n, x^{(1)}(n), \ldots, x^{(k)}(n)\right)\right\},
\end{aligned}
$$

$n \in \mathbb{N}_{0}$, where $k \in \mathbb{N}, l_{i} \in \mathbb{N}, i=\overline{1, k}, x^{(i)}(0) \in \mathbb{R}, i=\overline{1, k}$, and where the functions $f_{i j}: \mathbb{N}_{0} \times$ $\mathbb{R}^{k} \rightarrow \mathbb{R}, i=\overline{1, k}, j=\overline{1, l_{i}}$, are $\omega$-periodic in the first variable, that is, satisfy the equalities

$$
f_{i j}\left(n+\omega, u_{1}, \ldots, u_{k}\right)=f_{i j}\left(n, u_{1}, \ldots, u_{k}\right)
$$

for every $n \in \mathbb{N}_{0}$ and $u_{s} \in \mathbb{R}, s=\overline{1, k}$, and some $\omega \in \mathbb{N}$, and satisfy the following Lipschitztype conditions:

$$
\left|f_{i j}\left(n, u_{1}, \ldots, u_{k}\right)-f_{i j}\left(n, v_{1}, \ldots, v_{k}\right)\right| \leq \sum_{s=1}^{k} L_{i j s}\left|u_{s}-v_{s}\right|
$$

for every $n \in \mathbb{N}_{0}$ and $u_{s}, v_{s} \in \mathbb{R}, s=\overline{1, k}$, and for some $L_{i j s} \geq 0, i, s=\overline{1, k}, j=\overline{1, l_{l}}$, such that

$$
\widehat{q}:=\max _{1 \leq i \leq k, 1 \leq j \leq l_{i}} \sum_{s=1}^{k} L_{i j s}<1 .
$$

By $\mathbb{R}_{\infty}^{k}$, we will denote the $k$-dimensional real vector space with the following norm:

$$
\|\vec{a}\|_{\mathbb{R}_{\infty}^{k}}=\max _{i=1, k}\left|a_{i}\right|
$$

where $\vec{a}=\left(a_{1}, \ldots, a_{k}\right) \in \mathbb{R}^{k}$. 
A solution $(\vec{x}(n))_{n \in \mathbb{N}_{0}}=\left(x^{(1)}(n), \ldots, x^{(k)}(n)\right)_{n \in \mathbb{N}_{0}}$ to system (1) is called eventually periodic with period $\omega \in \mathbb{N}$ if there is $n_{0} \in \mathbb{N}$ such that

$$
x^{(i)}(n+\omega)=x^{(i)}(n)
$$

for every $n \geq n_{0}$ and $i=\overline{1, k}$ (see, e.g., $\left.[12,13,16]\right)$. If $n_{0}=0$, then such a solution to system (1) is called $\omega$-periodic.

Let $l_{\omega}^{\infty}\left(\mathbb{N}_{0}^{k}\right)$ be the space of all $\omega$-periodic vector sequences $x=(\vec{x}(n))_{n \in \mathbb{N}_{0}}=\left(x^{(1)}(n), \ldots\right.$, $\left.x^{(k)}(n)\right)_{n \in \mathbb{N}_{0}}$, with the following norm:

$$
\|x\|_{\omega_{\infty}}=\max _{0 \leq n \leq \omega-1}\|\vec{x}(n)\|_{\mathbb{R}_{\infty}^{k}}
$$

It is well known that $l_{\omega}^{\infty}\left(\mathbb{N}_{0}^{k}\right)$ with norm (5) is a Banach space.

Recently, in [2], which was the motivation for this paper of ours, it was shown that, under some more restrictive conditions than the ones proposed here, for the case $k=2$, max-type system (1) has a unique periodic solution with period $\omega$ and that every solution to the system converges to the periodic solution. A natural question is whether a related result holds for the case of $k$-dimensional system (1). In this paper we give a positive answer to the question. Moreover, we also present an elegant and considerably shorter proof of a more general result which includes the ones in [2].

One of the standard methods for showing the existence of a specific type of solutions of systems of difference equations is application of fixed point theorems. Various results of this type can be found, for example, in [22] and [23], and in the related references therein.

Assume that $(X, d)$ is a (non-empty) complete metric space and that operator $A: X \rightarrow X$ is a contraction, that is, the following inequality holds:

$$
d(A x, A y) \leq q d(x, y)
$$

for every $x, y \in X$ and some $q \in[0,1)$. The Banach fixed point theorem says that under these conditions operator $A$ has a unique fixed point, that is, a point $x^{*} \in X$ such that $A\left(x^{*}\right)=x^{*}$.

The idea in the paper is to define a natural operator on the metric (in fact, normed) space $\left(l_{\omega}^{\infty}\left(\mathbb{N}_{0}^{k}\right),\|\cdot\|_{\omega_{\infty}}\right)$, which will be a contraction, so that by using the Banach fixed point theorem can be shown the existence of a fixed point, which will be a unique periodic solution to system (1). For proving the global attractivity of all solutions to system (1) to the periodic one is essentially used a related stronger condition to (6), for which there is not needed any fixed point theorem, but just some estimates.

\section{Main result and some remarks}

In this section we prove the main result of this paper, and we also give some remarks related to the proof of the main result and conditions posed in it.

Theorem 1 Consider system (1), where $k \in \mathbb{N}, l_{i} \in \mathbb{N}, i=\overline{1, k}, x^{(i)}(0) \in \mathbb{R}, i=\overline{1, k}$, the functions $f_{i j}: \mathbb{N}_{0} \times \mathbb{R}^{k} \rightarrow \mathbb{R}, i=\overline{1, k}, j=\overline{1, l_{i}}$, satisfy conditions (2) and (3) with some nonnegative constants $L_{i j s}, i, s=\overline{1, k}, j=\overline{1, l_{i}}$, satisfying condition (4). Then system (1) has a unique $\omega$ periodic solution attracting all the solutions to the system. 
Proof For brevity, from now on we will write system (1) in the following form:

$$
x^{(i)}(n+1)=\max _{1 \leq j \leq l_{i}}\left\{f_{i j}(n, \vec{x}(n))\right\}, \quad n \in \mathbb{N}_{0}, i=\overline{1, k}
$$

Let $c$ be a real number such that

$$
0<c \leq \frac{1-\widehat{q}}{2}
$$

and write system (1) in the following form:

$$
x^{(i)}(n+1)=c x^{(i)}(n)+\max _{1 \leq j \leq l_{i}}\left\{f_{i j}(n, \vec{x}(n))-c x^{(i)}(n)\right\}
$$

for $n \in \mathbb{N}_{0}$ and $i=\overline{1, k}$.

On the family of all vector sequences, we define the operators $T_{i}, i=\overline{1, k}$, as follows:

$$
T_{i}(z)(n)=\max _{1 \leq j \leq l_{i}}\left\{f_{i j}(n, \vec{z}(n))-c z^{(i)}(n)\right\}
$$

for $n \in \mathbb{N}_{0}$ and $i=\overline{1, k}$.

Let

$$
T(z)(n)=\left(\sum_{s=1}^{\omega} \frac{c^{-s} T_{1}(z)(n+s-1)}{c^{-\omega}-1}, \ldots, \sum_{s=1}^{\omega} \frac{c^{-s} T_{k}(z)(n+s-1)}{c^{-\omega}-1}\right),
$$

for $n \in \mathbb{N}_{0}$.

Since the functions $f_{i j}, i=\overline{1, k}, j=\overline{1, l_{i}}$, are $\omega$-periodic in the first variable it immediately follows that $T_{i}(z)(n+\omega)=T_{i}(z)(n)$, for every $n \in \mathbb{N}_{0}$ and $i=\overline{1, k}$, and consequently one obtains

$$
T(z)(n+\omega)=T(z)(n), \quad n \in \mathbb{N}_{0},
$$

for every $z \in l_{\omega}^{\infty}\left(\mathbb{N}_{0}^{k}\right)$.

This means that the operator $T$ maps the Banach space $l_{\omega}^{\infty}\left(\mathbb{N}_{0}^{k}\right)$ into itself.

Now we estimate the following expressions:

$$
\left|T_{i}(x)(n)-T_{i}(y)(n)\right|=\left|\max _{1 \leq j \leq l_{i}}\left\{f_{i j}(n, \vec{x}(n))-c x^{(i)}(n)\right\}-\max _{1 \leq j \leq l_{i}}\left\{f_{i j}(n, \vec{y}(n))-c y^{(i)}(n)\right\}\right|,
$$

for $n \in \mathbb{N}_{0}$ and $i=\overline{1, k}$.

For each $i \in\{1, \ldots, k\}$, there are $j_{0}, j_{1} \in\left\{1, \ldots, l_{i}\right\}\left(j_{0}=j_{0}(i), j_{1}=j_{1}(i)\right)$, such that

$$
\max _{1 \leq j \leq l_{i}}\left\{f_{i j}(n, \vec{x}(n))\right\}=f_{i j_{0}}(n, \vec{x}(n))
$$

and

$$
\max _{1 \leq j \leq l_{i}}\left\{f_{i j}(n, \vec{y}(n))\right\}=f_{i j_{1}}(n, \vec{y}(n)),
$$


which implies

$$
\left|T_{i}(x)(n)-T_{i}(y)(n)\right|=\left|f_{i j_{0}}(n, \vec{x}(n))-c x^{(i)}(n)-f_{i j_{1}}(n, \vec{y}(n))+c y^{(i)}(n)\right| .
$$

If, for some $i \in\{1, \ldots, k\}$,

$$
f_{i j_{0}}(n, \vec{x}(n))-c x^{(i)}(n)-f_{i j_{1}}(n, \vec{y}(n))+c y^{(i)}(n) \geq 0,
$$

then from (12), the choice of $j_{1}$, and (3), we have

$$
\begin{aligned}
\left|T_{i}(x)(n)-T_{i}(y)(n)\right| & =f_{i j_{0}}(n, \vec{x}(n))-c x^{(i)}(n)-f_{i j_{1}}(n, \vec{y}(n))+c y^{(i)}(n) \\
& \leq f_{i j_{0}}(n, \vec{x}(n))-c x^{(i)}(n)-f_{i j_{0}}(n, \vec{y}(n))+c y^{(i)}(n) \\
& \leq \sum_{s=1}^{k} L_{i j_{0} s}\left|x^{(s)}(n)-y^{(s)}(n)\right|+c\left|x^{(i)}(n)-y^{(i)}(n)\right| \\
& \leq\left(c+\sum_{s=1}^{k} L_{i j_{0} s}\right)\|\vec{x}(n)-\vec{y}(n)\|_{\mathbb{R}_{\infty}^{k}} .
\end{aligned}
$$

On the other hand, if for some $i \in\{1, \ldots, k\}$,

$$
f_{i j_{0}}(n, \vec{x}(n))-c x^{(i)}(n)-f_{i j_{1}}(n, \vec{y}(n))+c y^{(i)}(n) \leq 0,
$$

then from (12), the choice of $j_{0}$, and (3), we have

$$
\begin{aligned}
\left|T_{i}(x)(n)-T_{i}(y)(n)\right| & =f_{i j_{1}}(n, \vec{y}(n))-c y^{(i)}(n)-f_{i j_{0}}(n, \vec{x}(n))+c x^{(i)}(n) \\
& \leq f_{i j_{1}}(n, \vec{y}(n))-c y^{(i)}(n)-f_{i j_{1}}(n, \vec{x}(n))+c x^{(i)}(n) \\
& \leq \sum_{s=1}^{k} L_{i j_{1} s}\left|x^{(s)}(n)-y^{(s)}(n)\right|+c\left|x^{(i)}(n)-y^{(i)}(n)\right| \\
& \leq\left(c+\sum_{s=1}^{k} L_{i j_{1} s}\right)\|\vec{x}(n)-\vec{y}(n)\|_{\mathbb{R}_{\infty}^{k}} .
\end{aligned}
$$

From (4), (13), and (14) it follows that

$$
\left|T_{i}(x)(n)-T_{i}(y)(n)\right| \leq(c+\widehat{q})\|\vec{x}(n)-\vec{y}(n)\|_{\mathbb{R}_{\infty}^{k}}
$$

for every $n \in \mathbb{N}_{0}$ and $i=\overline{1, k}$.

Using (11) and (15), we have

$$
\begin{aligned}
\|T(x)(n)-T(y)(n)\|_{\mathbb{R}_{\infty}^{k}} & =\max _{i=1, k}\left|\sum_{s=1}^{\omega} \frac{c^{-s}\left(T_{i}(x)(n+s-1)-T_{i}(y)(n+s-1)\right)}{c^{-\omega}-1}\right| \\
& \leq(c+\widehat{q}) \sum_{s=1}^{\omega} \frac{c^{-s}}{c^{-\omega}-1}\|\vec{x}(n)-\vec{y}(n)\|_{\mathbb{R}_{\infty}^{k}} \\
& =\frac{c+\widehat{q}}{1-c}\|\vec{x}(n)-\vec{y}(n)\|_{\mathbb{R}_{\infty}^{k}}
\end{aligned}
$$

for every $n \in \mathbb{N}_{0}$ and $x, y \in l_{\omega}^{\infty}\left(\mathbb{N}_{0}^{k}\right)$. 
By taking the supremum in (16) over the set $\{n: 0 \leq n \leq \omega-1\}$, we get

$$
\|T x-T y\|_{\omega_{\infty}} \leq q_{1}\|x-y\|_{\omega_{\infty}}
$$

for every $x, y \in l_{\omega}^{\infty}\left(\mathbb{N}_{0}^{k}\right)$, where

$$
q_{1}:=\frac{c+\widehat{q}}{1-c}<1
$$

due to condition (8).

This means that the operator $T$ is a contraction on $l_{\omega}^{\infty}\left(\mathbb{N}_{0}^{k}\right)$. Hence, by the Banach fixed point theorem it follows that there is a unique fixed point $z \in l_{\omega}^{\infty}\left(\mathbb{N}_{0}^{k}\right)$ of $T$, that is,

$$
z^{(i)}(n)=\sum_{s=1}^{\omega} \frac{c^{-s} T_{i}(z)(n+s-1)}{c^{-\omega}-1}, \quad n \in \mathbb{N}_{0}, i=\overline{1, k}
$$

holds. By using (18), some calculation and $\omega$-periodicity of $z$, it is not difficult to see that $z$ is also a solution to system (1), which proves the first statement in the theorem.

Let $x$ be a solution to system (1) and $z$ be the $\omega$-periodic solution to the system, then by using the change of variables $y=x-z$ the system becomes

$$
y^{(i)}(n+1)=\max _{1 \leq j \leq l_{i}}\left\{f_{i j}(n, \vec{y}(n)+\vec{z}(n))\right\}-\max _{1 \leq j \leq l_{i}}\left\{f_{i j}(n, \vec{z}(n))\right\}
$$

for every $n \in \mathbb{N}_{0}$ and $i=\overline{1, k}$.

For each $i \in\{1, \ldots, k\}$, there are $j_{2}, j_{3} \in\{1, \ldots, k\}\left(j_{2}=j_{2}(i), j_{3}=j_{3}(i)\right)$, such that

$$
\max _{1 \leq j \leq l_{i}}\left\{f_{i j}(n, \vec{y}(n)+\vec{z}(n))\right\}=f_{i j_{2}}(n, \vec{y}(n)+\vec{z}(n))
$$

and

$$
\max _{1 \leq j \leq l_{i}}\left\{f_{i j}(n, \vec{z}(n))\right\}=f_{i j_{3}}(n, \vec{z}(n))
$$

which implies

$$
y^{(i)}(n+1)=f_{i j_{2}}(n, \vec{y}(n)+\vec{z}(n))-f_{i j_{3}}(n, \vec{z}(n)) .
$$

If for some $i \in\{1, \ldots, k\}$,

$$
f_{i j_{2}}(n, \vec{y}(n)+\vec{z}(n))-f_{i j_{3}}(n, \vec{z}(n)) \geq 0
$$

then from (20), the choice of $j_{3}$ and (3), we have

$$
\begin{aligned}
\left|y^{(i)}(n+1)\right| & =f_{i j_{2}}(n, \vec{y}(n)+\vec{z}(n))-f_{i j_{3}}(n, \vec{z}(n)) \\
& \leq f_{i j_{2}}(n, \vec{y}(n)+\vec{z}(n))-f_{i j_{2}}(n, \vec{z}(n)) \\
& \leq \sum_{s=1}^{k} L_{i j_{2} s}\left|y^{(s)}(n)\right| \leq\left(\sum_{s=1}^{k} L_{i j_{2} s}\right)\|\vec{y}(n)\|_{\mathbb{R}_{\infty}^{k}} .
\end{aligned}
$$


On the other hand, if, for some $i \in\{1, \ldots, k\}$,

$$
f_{i j_{2}}(n, \vec{y}(n)+\vec{z}(n))-f_{i j_{3}}(n, \vec{z}(n)) \leq 0,
$$

then from (20), the choice of $j_{2}$, and (3), we have

$$
\begin{aligned}
\left|y^{(i)}(n+1)\right| & =f_{i j_{3}}(n, \vec{z}(n))-f_{i j_{2}}(n, \vec{y}(n)+\vec{z}(n)) \\
& \leq f_{i j_{3}}(n, \vec{z}(n))-f_{i j_{3}}(n, \vec{y}(n)+\vec{z}(n)) \\
& \leq \sum_{s=1}^{k} L_{i j_{3} s}\left|y^{(s)}(n)\right| \\
& \leq\left(\sum_{s=1}^{k} L_{i j_{3} s}\right)\|\vec{y}(n)\|_{\mathbb{R}_{\infty}^{k}} .
\end{aligned}
$$

From (21) and (22) it easily follows that

$$
\|\vec{y}(n+1)\|_{\mathbb{R}_{\infty}^{k}} \leq \widehat{q}\|\vec{y}(n)\|_{\mathbb{R}_{\infty}^{k}}, \quad n \in \mathbb{N}_{0},
$$

from which one obtains

$$
\|\vec{y}(n)\|_{\mathbb{R}_{\infty}^{k}} \leq \widehat{q}^{n}\|\vec{y}(0)\|_{\mathbb{R}_{\infty}^{k}}, \quad n \in \mathbb{N}_{0}
$$

From (23) and the fact $\widehat{q} \in[0,1)$, it follows that

$$
\lim _{n \rightarrow \infty}\|\vec{y}(n)\|_{\mathbb{R}_{\infty}^{k}}=0
$$

from which the second statement of the theorem follows, finishing the proof of the theorem.

Remark 1 If for some fixed $i, j \in\{1, \ldots, k\}$ the corresponding function $f_{i j}$ does not contain all variables $u_{1}, u_{2}, \ldots, u_{k}$, but only some of them, say $u_{1}, \ldots, u_{m}$, where $m<k$, then the function $f_{i j}$ which maps the set $\mathbb{N}_{0} \times \mathbb{R}^{m}$ to $\mathbb{R}$ can be regarded, in a natural way, as a function from the set $\mathbb{N}_{0} \times \mathbb{R}^{k}$ into $\mathbb{R}$, by using the following extension:

$$
\tilde{f}_{i j}\left(n, u_{1}, \ldots, u_{k}\right)=f_{i j}\left(n, u_{1}, \ldots, u_{m}\right)
$$

for every $n \in \mathbb{N}_{0}, u_{i} \in \mathbb{R}, i=\overline{1, k}$.

Such a situation appears in [2] where all the involved functions are defined on the set $\mathbb{N}_{0} \times \mathbb{R}$, but they can be naturally prolonged on the set $\mathbb{N}_{0} \times \mathbb{R}^{2}$ as suggested above.

Remark 2 It is interesting to note that we cannot choose $c=0$ in the proof of the first statement in Theorem 1. Namely, if we do this, then the operators $T_{i}, i=\overline{1, k}$, become

$$
T_{i}(z)(n)=\max _{1 \leq j \leq l_{i}}\left\{f_{i j}(n, \vec{z}(n))\right\}, \quad n \in \mathbb{N}_{0}, i=\overline{1, k}
$$


and a natural choice for the operator $T$ is

$$
T=\left(T_{1}, \ldots, T_{k}\right) .
$$

From the proof of Theorem 1 with $c=0$, for $T$ such chosen, it is easily seen that (see (15))

$$
\|T(x)-T(y)\|_{\omega_{\infty}} \leq \hat{q}\|x-y\|_{\omega_{\infty}}
$$

for every $x, y \in l_{\omega}^{\infty}\left(\mathbb{N}_{0}^{k}\right)$.

Hence, the operator $T: l_{\omega}^{\infty}\left(\mathbb{N}_{0}^{k}\right) \rightarrow l_{\omega}^{\infty}\left(\mathbb{N}_{0}^{k}\right)$ is a contraction. By the Banach fixed point theorem it follows that there is a unique $z \in l_{\omega}^{\infty}\left(\mathbb{N}_{0}^{k}\right)$ such that $T(z)=z$, that is,

$$
z^{(i)}(n+1)=z^{(i)}(n)
$$

for every $n \in \mathbb{N}_{0}$ and $i=\overline{1, k}$, which is, in fact, the existence of a constant vector solution to $(1)$.

Let $(\vec{c})_{n \in \mathbb{N}_{0}}=\left(\left(c_{1}, \ldots, c_{k}\right)\right)_{n \in \mathbb{N}_{0}}$ be a constant solution to (1). Then for each $i \in\{1, \ldots, k\}$ there is a $j_{i} \in\left\{1, \ldots, l_{i}\right\}$ such that

$$
c_{i}=f_{i j i}(n, \vec{c}) \text {, }
$$

for every $n \in \mathbb{N}_{0}$, from which it follows that the values of functions $f_{i j_{i}}$ are independent of $n$. Note that due to the periodicity of $f_{i i_{i}}$ the variable $n$ can essentially take only the values from the set $\{0,1, \ldots, \omega-1\}$. This suggests that the following closely related result, which could be known, holds.

\section{Proposition 1 Consider the system of equations}

$$
c_{i}=\max _{1 \leq j \leq l_{i}}\left\{g_{i j}(\vec{c})\right\}, \quad i=\overline{1, k}
$$

where $k \in \mathbb{N}, l_{i} \in \mathbb{N}, i=\overline{1, k}$, the functions $g_{i j}: \mathbb{R}^{k} \rightarrow \mathbb{R}, i=\overline{1, k}, j=\overline{1, l_{i}}$, satisfy the following Lipschitz-type conditions:

$$
\left|g_{i j}\left(u_{1}, \ldots, u_{k}\right)-g_{i j}\left(v_{1}, \ldots, v_{k}\right)\right| \leq \sum_{s=1}^{k} \widehat{L}_{i j s}\left|u_{s}-v_{s}\right|
$$

for every $u_{s}, v_{s} \in \mathbb{R}, s=\overline{1, k}$, and for some nonnegative numbers $\widehat{L}_{i j j}, i, s=\overline{1, k}, j=\overline{1, l_{i}}$, such that

$$
q_{1}:=\max _{1 \leq i \leq k, 1 \leq j \leq l_{i}} \sum_{s=1}^{k} \widehat{L}_{i j s}<1
$$

Then system (24) has a unique solution in $\mathbb{R}^{k}$. 
Proof Let

$$
\widehat{M}:=\max _{1 \leq i \leq k, 1 \leq j \leq l_{i}}\left|g_{i j}(\overrightarrow{0})\right|,
$$

and the operator $T=\left(T_{1}, \ldots, T_{k}\right)$ be defined on $\mathbb{R}^{k}$ as follows:

$$
T(\vec{u})=\left(\max _{1 \leq j \leq l_{1}}\left\{g_{1 j}(\vec{u})\right\}, \ldots, \max _{1 \leq j \leq l_{k}}\left\{g_{k j}(\vec{u})\right\}\right) .
$$

Then by using (25) and (26), we have

$$
\begin{aligned}
\|T(\vec{u})\|_{\mathbb{R}_{\infty}^{k}} & =\max _{i=\overline{1, k}}\left|\max _{1 \leq j \leq l_{i}}\left\{g_{i j}(\vec{u})\right\}\right| \leq \max _{1 \leq i \leq k, 1 \leq j \leq l_{i}}\left\{\left|g_{i j}(\overrightarrow{0})\right|+\left|g_{i j}(\vec{u})-g_{i j}(\overrightarrow{0})\right|\right\} \\
& \leq \max _{1 \leq i \leq k, 1 \leq j \leq l_{i}}\left\{\widehat{M}+\sum_{s=1}^{k} \widehat{L}_{i j s}\left|u_{s}\right|\right\} \\
& \leq \widehat{M}+q_{1}\|\vec{u}\|_{\mathbb{R}_{\infty}^{k}} .
\end{aligned}
$$

Let

$$
r=\frac{\widehat{M}}{1-q_{1}} .
$$

Then from (27) it follows that $T$ maps the closed ball $B_{r}=\left\{\vec{u} \in \mathbb{R}^{k}:\|\vec{u}\|_{\mathbb{R}_{\infty}^{k}} \leq r\right\}$ into itself.

On the other hand, similar to the proof of Theorem 1 , one obtains

$$
\|T(\vec{u})-T(\vec{v})\|_{\mathbb{R}_{\infty}^{k}} \leq q_{1}\|\vec{u}-\vec{v}\|_{\mathbb{R}_{\infty}^{k}}
$$

for every $\vec{u}, \vec{v} \in \mathbb{R}^{k}$, that is, $T: B_{r} \rightarrow B_{r}$ is a contraction.

Hence, by the Banach fixed point theorem it follows that there is a unique fixed point of the operator, that is, a unique solution to system (24) in $B_{r}$. Due to (28) it is also a unique solution in $\mathbb{R}^{k}$.

\section{Competing interests}

The author declares that he has no competing interests.

\section{Author's contributions}

The author contributed alone to the writing of this paper. He read and approved the manuscript.

Received: 7 September 2016 Accepted: 20 September 2016 Published online: 29 September 2016

\section{References}

1. Berenhaut, K, Foley, J, Stević, S: Boundedness character of positive solutions of a max difference equation. J. Differ. Equ. Appl. 12(12), 1193-1199 (2006)

2. Dekkar, I, Touafek, N: Existence and global attractivity of periodic solutions in a max-type system of difference equations. Turk. J. Math. (2016, in press)

3. Iričanin, BD, Touafek, N: On a second-order max-type system of difference equations. Indian J. Math. 54(1), 119-142 (2012)

4. Kent, CM, Kustesky, M, Nguyen, $A Q$, Nguyen, BV: Eventually periodic solutions of $x_{n+1}=\max \left\{A_{n} / x_{n}, B_{n} / x_{n-1}\right\}$ when the parameters are two cycles. Dyn. Contin. Discrete Impuls. Syst., Ser. A Math. Anal. 10(1-3), 33-49 (2003)

5. Liu, W, Stević, S: Global attractivity of a family of nonautonomous max-type difference equations. Appl. Math. Comput. 218, 6297-6303 (2012)

6. Papaschinopoulos, G, Hatzifilippidis, V: On a max difference equation. J. Math. Anal. Appl. 258, $258-268$ (2001)

7. Papaschinopoulos, G, Schinas, C, Hatzifilippidis, V: Global behavior of the solutions of a max-equation and of a system of two max-equations. J. Comput. Anal. Appl. 5, 237-254 (2003) 
8. Stefanidou, G, Papaschinopoulos, G, Schinas, C: On a system of max difference equations. Dyn. Contin. Discrete Impuls. Syst., Ser. A Math. Anal. 14(6), 885-903 (2007)

9. Stević, S: Boundedness character of a class of difference equations. Nonlinear Anal. TMA 70, 839-848 (2009)

10. Stević, S: Global stability of a difference equation with maximum. Appl. Math. Comput. 210, 525-529 (2009)

11. Stević, S: On a generalized max-type difference equation from automatic control theory. Nonlinear Anal. TMA 72, 1841-1849 (2010)

12. Stević, S: Periodicity of max difference equations. Util. Math. 83, 69-71 (2010)

13. Stević, S: On some periodic systems of max-type difference equations. Appl. Math. Comput. 218, 11483-11487 (2012)

14. Stević, S: On positive solutions of some classes of max-type systems of difference equations. Appl. Math. Comput. 232C, 445-452 (2014)

15. Stević, S, Alghamdi, MA, Alotaibi, A, Shahzad, N: Boundedness character of a max-type system of difference equations of second order. Electron. J. Qual. Theory Differ. Equ. 2014, Article ID 45 (2014)

16. Stević, S, Alghamdi, MA, Alotaibi, A, Shahzad, N: Eventual periodicity of some systems of max-type difference equations. Appl. Math. Comput. 236C, 635-641 (2014)

17. Sun, $T, X i, H, H a n, C, Q i n, B$ : Dynamics of the max-type difference equation $x_{n}=\max \left\{1 / x_{n-m}, A_{n} / x_{n-r}\right\}$. J. Appl. Math. Comput. 38(1-2), 173-180 (2012)

18. Papaschinopoulos, G, Schinas, CJ: On the behavior of the solutions of a system of two nonlinear difference equations. Commun. Appl. Nonlinear Anal. 5(2), 47-59 (1998)

19. Papaschinopoulos, G, Schinas, CJ: Invariants for systems of two nonlinear difference equations. Differ. Equ. Dyn. Syst. 7(2), 181-196 (1999)

20. Stević, S: Solutions of a max-type system of difference equations. Appl. Math. Comput. 218, 9825-9830 (2012)

21. Stević, S, Diblik, J, Iričanin, B, Šmarda, Z: Solvability of nonlinear difference equations of fourth order. Electron. J. Differ. Equ. 2014, Article ID 264 (2014)

22. Agarwal, RP: Difference Equations and Inequalities: Theory, Methods, and Applications, 2nd edn. Dekker, New York (2000)

23. Diblik, J, Schmeidel, E: On the existence of solutions of linear Volterra difference equations asymptotically equivalent to a given sequence. Appl. Math. Comput. 218, 9310-9320 (2012)

\section{Submit your manuscript to a SpringerOpen ${ }^{\ominus}$ journal and benefit from:}

- Convenient online submission

Rigorous peer review

Immediate publication on acceptance

- Open access: articles freely available online

- High visibility within the field

- Retaining the copyright to your article 